Pacific

Journal of

Mathematics

\author{
ON KRONECKER PRODUCTS \\ OF COMPLEX REPRESENTATIONS \\ OF THE SYMMETRIC AND ALTERNATING GROUPS
}

C. Bessenrodt and A. Kleshchev 


\title{
ON KRONECKER PRODUCTS OF COMPLEX REPRESENTATIONS OF THE SYMMETRIC AND ALTERNATING GROUPS
}

\author{
C. Bessenrodt and A. Kleshchev
}

\begin{abstract}
In this paper we study the homogeneous tensor products of simple modules over symmetric and alternating groups.
\end{abstract}

\section{Introduction.}

Kronecker or inner tensor products of representations of symmetric groups (and many other groups) have been studied for a long time. But even for the symmetric groups no reasonable formula for decomposing Kronecker products of two irreducible complex representations into irreducible components is available (cf. $[\mathbf{7}, \mathbf{5}]$ ). An equivalent problem is to decompose the inner product of the corresponding Schur functions into a linear combination of Schur functions.

In recent years, a number of partial results have been obtained. For example, the products of characters labelled by hook partitions or by two-row partitions $[3,8]$ have been computed, and special constituents, in particular of tensor squares, have been considered $[\mathbf{1 0}, \mathbf{1 1}, \mathbf{1 2}]$. For general products, Dvir [2] and Clausen-Meier [1] determined the largest part and the maximal number of parts in a constituent of a product (this result is crucial in this paper).

In general, Kronecker products of irreducible representations have very many irreducible constituents (see e.g. $[4,2.9]$ ). In this paper, we first consider the simple question: 'when is the Kronecker product of two irreducible $S_{n}$-characters again irreducible?' We prove that in fact such a product is always reducible, and even inhomogeneous, except for the obvious exception where one of the characters is of degree 1 . Then we turn to the same question for the representations of the alternating group $A_{n}$. Here one can easily construct examples of non-trivial irreducible tensor products (actually, we observed this first using calculations with the MAPLE packages SF (by Stembridge) and ACE (by Veigneau et al.)). It turns out that the problem for $A_{n}$ reduces to the classification of certain products of $S_{n}$-characters with 2 constituents. So we classify in general the Kronecker products of $S_{n}$-characters with 2 constituents, and even more generally, with two homogeneous components. We also obtain some partial results for products with 
4 homogeneous components and conjecture a complete classification of the pairs $\left(L_{1}, L_{2}\right)$ of irreducible complex $S_{n}$-representations such that $L_{1} \otimes L_{2}$ has at most 4 homogeneous components.

\section{Preliminaries.}

We denote by $\mathbb{N}$ the set $\{1,2, \ldots\}$ of the natural numbers.

If $G$ and $H$ are two groups, $L$ is a $\mathbb{C} G$-module and $M$ is a $\mathbb{C} H$-module we write $L \otimes M$ for the outer tensor product of $L$ and $M$ (which is a module over $G \times H)$. If $N$ is another $\mathbb{C} G$-module we write $L \otimes N$ for the inner tensor (or Kronecker) product of $L$ and $N$ (which is a $G$-module).

A $\mathbb{C} G$-module is called homogeneous if it is isomorphic to a direct sum of copies of one simple module. Every $\mathbb{C} G$-module can be (uniquely) decomposed into a direct sum of its homogeneous components. Similarly we speak of the homogeneous characters and the homogeneous components of the characters.

We use the notions and notation of the representation theory of $S_{n}$ and $A_{n}$ and refer the reader to [4] for the most basic ones. In particular, we write $\lambda=\left(\lambda_{1}, \ldots, \lambda_{k}\right) \vdash n$ if $\lambda$ is a partition of $n$; in this case we also write $|\lambda|$ for $n$. We often gather together equal parts of a partition and write, for example, $\left(5^{2}, 3^{3}\right)$ for $(5,5,3,3,3)$. The partition conjugate to $\lambda$ is denoted by $\lambda^{\prime}$. If $\lambda=\lambda^{\prime}$ we say that $\lambda$ is symmetric. We do not distinguish between a partition $\lambda$ and its Young diagram $\lambda=\left\{(i, j) \in \mathbb{N} \times \mathbb{N} \mid j \leq \lambda_{i}\right\}$. Elements $(i, j) \in \mathbb{N} \times \mathbb{N}$ are called nodes. If $\lambda=\left(\lambda_{1}, \lambda_{2}, \ldots\right)$ and $\mu=\left(\mu_{1}, \mu_{2}, \ldots\right)$ are two partitions we write $\lambda \cap \mu$ for the partition $\left(\min \left(\lambda_{1}, \mu_{1}\right), \min \left(\lambda_{2}, \mu_{2}\right), \ldots\right)$ whose Young diagram is just the intersection of those for $\lambda$ and $\mu$. A node $\left(i, \lambda_{i}\right) \in \lambda$ is called removable (for $\lambda$ ) if $\lambda_{i}>\lambda_{i+1}$. A node $\left(i, \lambda_{i}+1\right)$ is called addable (for $\lambda$ ) if $i=1$ or $i>1$ and $\lambda_{i}<\lambda_{i-1}$. We denote by

$$
\lambda_{A}=\lambda \backslash\{A\}=\left(\lambda_{1}, \ldots, \lambda_{i-1}, \lambda_{i}-1, \lambda_{i+1}, \ldots\right)
$$

a partition of $n-1$ obtained by removing a removable node $A=\left(i, \lambda_{i}\right)$ from $\lambda$. Similarly

$$
\lambda^{B}=\lambda \cup\{B\}=\left(\lambda_{1}, \ldots, \lambda_{i-1}, \lambda_{i}+1, \lambda_{i+1}, \ldots\right)
$$

is a partition of $n+1$ obtained by adding an addable node $B=\left(i, \lambda_{i}+1\right)$ to $\lambda$.

We denote by

$$
h_{i j}=h_{i j}^{\lambda}=\lambda_{i}-j+\lambda_{j}^{\prime}-i+1
$$

the $(i, j)$-hook length. If a partition $\lambda$ has $r$ nodes on the main diagonal and there are $\alpha_{i}$ (resp., $\beta_{i}$ ) nodes to the right of (resp., below) the node $(i, i)$ then we may write $\lambda$ in the Frobenius notation (cf. [6]):

$$
F(\lambda)=\left(\begin{array}{ccc}
\alpha_{1} & \cdots & \alpha_{r} \\
\beta_{1} & \cdots & \beta_{r}
\end{array}\right)
$$


If $H_{\lambda} \cong S_{\lambda_{1}} \times S_{\lambda_{2}} \times \cdots<S_{n}$ is a Young subgroup we write $M^{\lambda}$ for the permutation module $\mathbb{C} S_{n} \otimes_{\mathbb{C} H_{\lambda}} 1_{H_{\lambda}}$. The Specht module $S^{\lambda}$ is explicitly defined as a submodule of $M^{\lambda}$ (cf. [4]). The set $\left\{S^{\lambda} \mid \lambda \vdash n\right\}$ is a complete set of irreducible $\mathbb{C} S_{n}$-modules (up to isomorphism). We write $[\lambda]$ (or $\left.\left[\lambda_{1}, \lambda_{2}, \ldots\right]\right)$ for the character of $S^{\lambda}$. Thus, $\{[\lambda] \mid \lambda \vdash n\}$ is a complete set of the irreducible characters of $S_{n}$. It is well known that $S^{\lambda}$ is self-dual. Another fact (to be used without comment) is that $S^{\left(1^{n}\right)}$ is the 1-dimensional sign representation and $S^{\lambda} \otimes S^{\left(1^{n}\right)} \cong S^{\lambda^{\prime}}$. The standard inner product on the class functions on a group (symmetric or alternating, depending on the context) is denoted by $\langle\cdot, \cdot\rangle$. If $\chi$ and $\psi$ are two class functions we write $\chi \cdot \psi$ for the function $[g \mapsto \chi(g) \psi(g)]$. The character of $S^{\lambda} \otimes S^{\mu}$ is $[\lambda] \cdot[\mu]$. For $\lambda, \mu, \nu \vdash n$ we define the numbers $d(\mu, \nu ; \lambda)$ via

$$
[\mu] \cdot[\nu]=\sum_{\lambda} d(\mu, \nu ; \lambda)[\lambda] .
$$

If $\alpha=\left(\alpha_{1}, \alpha_{2}, \ldots\right)$ and $\beta=\left(\beta_{1}, \beta_{2}, \ldots\right)$ are two partitions then we write $\beta \subseteq \alpha$ if $\beta_{i} \leq \alpha_{i}$ for all $i$. In this case we also consider the skew partition $\alpha / \beta$. We do not distinguish between $\alpha / \beta$ and its Young diagram, which is the set of nodes $\alpha \backslash \beta$.

If $\alpha / \beta$ is a skew Young diagram and $A=(i, j)$ is some node we say $A$ is connected with $\alpha / \beta$ if at least one of the nodes $(i \pm 1, j),(i, j \pm 1)$ belongs to $\alpha / \beta$. Otherwise $A$ is disconnected from $\alpha / \beta$.

If $\beta \vdash m, \gamma \vdash n, \alpha \vdash m+n$ we write $c_{\beta \gamma}^{\alpha}$ for the corresponding LittlewoodRichardson coefficient, which may be defined as the multiplicity of $S^{\alpha}$ in the induced module

$$
S^{\beta} \hat{\otimes} S^{\gamma}:=\left(S^{\beta} \otimes S^{\gamma}\right) \uparrow_{S_{m} \times S_{n}}^{S_{m+n}} .
$$

The character of this module will be denoted $[\beta] \hat{\otimes}[\gamma]$. The LittlewoodRichardson rule $[4,6]$ gives a combinatorial description of the coefficients $c_{\beta \gamma}^{\alpha}$ and will be repeatedly used in this paper. It says that $c_{\beta \gamma}^{\alpha}$ is the number of semistandard tableaux of skew shape $\alpha / \beta$ and content $\gamma$, which give a lattice permutation when the entries are read from right to left along the rows starting from the top row.

Let $\alpha$ and $\beta$ be two partitions. Then the skew character $[\alpha / \beta]$ is defined to be the sum

$$
[\alpha / \beta]=\sum_{\gamma} c_{\beta \gamma}^{\alpha}[\gamma]
$$

Note that $[\alpha / \beta]=0$ unless $\beta \subseteq \alpha$.

The following four results will be used repeatedly.

Theorem 2.1 ([2, 1.6], $[\mathbf{1}, 1.1])$. Let $\mu, \nu$ be partitions of $n$. Then

$$
\max \left\{\lambda_{1} \mid d(\mu, \nu ; \lambda) \neq 0 \text { for some } \lambda=\left(\lambda_{1}, \lambda_{2}, \ldots\right)\right\}=|\mu \cap \nu|
$$


and

$$
\max \left\{m \mid d(\mu, \nu ; \lambda) \neq 0 \text { for some } \lambda=\left(\lambda_{1} \geq \cdots \geq \lambda_{m}>0\right)\right\}=\left|\mu \cap \nu^{\prime}\right| .
$$

Since the skew characters can in principle be decomposed into the irreducible characters, the following theorem provides a recursive formula for the coefficients $d(\mu, \nu ; \lambda)$.

Theorem $2.2([2,2.3])$. Let $\mu, \nu$ and $\lambda=\left(\lambda_{1}, \lambda_{2}, \ldots\right)$ be partitions of $n$, and set $\hat{\lambda}=\left(\lambda_{2}, \lambda_{3}, \ldots\right)$. Define

$$
Y(\lambda)=\left\{\eta \mid \eta \vdash n, \eta_{i} \geq \lambda_{i+1} \geq \eta_{i+1} \text { for all } i \geq 1\right\} \text {. }
$$

Then

$$
d(\mu, \nu ; \lambda)=\sum_{\substack{\alpha \vdash \lambda_{1} \\ \alpha \subseteq \mu \cap \nu}}\langle[\mu / \alpha] \cdot[\nu / \alpha],[\hat{\lambda}]\rangle-\sum_{\substack{\eta \in Y(\lambda) \\ \eta \neq \lambda \\ \eta \neq \lambda}} d(\mu, \nu ; \eta) .
$$

Corollary $2.3([2,2.4],[\mathbf{1}, 2.1(\mathrm{~d})])$. Let $\mu, \nu$ and $\lambda=\left(\lambda_{1}, \lambda_{2}, \ldots\right)$ be partitions of $n$, and set $\hat{\lambda}=\left(\lambda_{2}, \lambda_{3}, \ldots\right), \gamma=\mu \cap \nu$. Assume that $\lambda_{1}=|\mu \cap \nu|$. Then

$$
d(\mu, \nu ; \lambda)=\langle[\mu / \gamma] \cdot[\nu / \gamma],[\hat{\lambda}]\rangle .
$$

Corollary $2.4\left(\left[2,2.4^{\prime}\right]\right)$. Let $\mu$ and $\nu$ be partitions of $n$, and $m=\left|\mu \cap \nu^{\prime}\right|$. Let $\lambda$ be a partition of $n$ with $m$ non-zero parts. Define $\bar{\lambda}=\left(\lambda_{1}-1, \lambda_{2}-\right.$ $\left.1, \ldots, \lambda_{m}-1\right)$. Then

$$
d(\mu, \nu ; \lambda)=\left\langle\left[\mu /\left(\mu \cap \nu^{\prime}\right)\right] \cdot\left[\nu /\left(\mu^{\prime} \cap \nu\right)\right],[\bar{\lambda}]\right\rangle .
$$

\section{Homogenous $S_{n}$-products.}

Lemma 3.1. Let $\alpha, \beta, a, b$ be positive integers. Then

$$
\min (\alpha+\beta+1, a+b+1)<\min (\alpha, a)+\min (\beta, b)+\min (\alpha, b)+\min (\beta, a) .
$$

Proof. We may assume that $\alpha \leq \beta, a \leq b$ and $\alpha+\beta \leq a+b$. So the left hand side in (1) is $\alpha+\beta+1$.

If $\beta \leq b$, then the right hand side of (1) equals

$$
\min (\alpha, a)+\beta+\alpha+\min (\beta, a)
$$

which is greater than $\alpha+\beta+1$ since all numbers in this expression are positive integers.

If $b<\beta$, then the right hand side of (1) is $\min (\alpha, a)+b+\min (\alpha, b)+a \geq \min (\alpha, a)+\min (\alpha, b)+\alpha+\beta>\alpha+\beta+1$, as claimed.

Lemma 3.2. Let $\mu, \nu$ be partitions of $n$, both different from $(n)$ and $\left(1^{n}\right)$. Then

$$
\min \left(h_{11}^{\mu}, h_{11}^{\nu}\right)<|\mu \cap \nu|+\left|\mu \cap \nu^{\prime}\right|-2 .
$$


Proof. We write $\mu$ and $\nu$ in the Frobenius notation:

$$
F(\mu)=\left(\begin{array}{ccc}
\alpha_{1} & \cdots & \alpha_{r} \\
\beta_{1} & \cdots & \beta_{r}
\end{array}\right), \quad F(\nu)=\left(\begin{array}{ccc}
a_{1} & \cdots & a_{s} \\
b_{1} & \cdots & b_{s}
\end{array}\right) .
$$

We may assume that $r \leq s$. Then

$$
\begin{gathered}
h_{11}^{\mu}=\alpha_{1}+\beta_{1}+1, \quad h_{11}^{\nu}=a_{1}+b_{1}+1, \\
|\mu \cap \nu|=r+\sum_{i=1}^{r}\left(\min \left(\alpha_{i}, a_{i}\right)+\min \left(\beta_{i}, b_{i}\right)\right), \\
\left|\mu \cap \nu^{\prime}\right|=r+\sum_{i=1}^{r}\left(\min \left(\alpha_{i}, b_{i}\right)+\min \left(\beta_{i}, a_{i}\right)\right) .
\end{gathered}
$$

Since $r \geq 1$, it suffices to prove that

$$
\begin{aligned}
\min \left(\alpha_{1}+\beta_{1}+\right. & \left.1, a_{1}+b_{1}+1\right) \\
& <\min \left(\alpha_{1}, a_{1}\right)+\min \left(\beta_{1}, b_{1}\right)+\min \left(\alpha_{1}, b_{1}\right)+\min \left(\beta_{1}, a_{1}\right) .
\end{aligned}
$$

But this follows from Lemma 3.1 since our assumption on the partitions ensures that $\alpha_{1}, \beta_{1}, a_{1}, b_{1}>0$.

Theorem 3.3. Let $\mu, \nu$ be partitions of $n$, both different from $(n)$ and $\left(1^{n}\right)$. If $[\lambda]$ is a constituent of $[\mu] \cdot[\nu]$, then $h_{11}^{\lambda}<|\mu \cap \nu|+\left|\mu \cap \nu^{\prime}\right|-1$.

Proof. Put $\ell=|\mu \cap \nu|+\left|\mu \cap \nu^{\prime}\right|-1$. Take $\pi$ to be an $\ell$-cycle in $S_{n}$. By Lemma 3.2, either $\mu$ or $\nu$ does not have a hook of length $\ell$. Hence, by the Murnaghan-Nakayama Rule $[4,2.4 .7]$, either $[\mu](\pi)=0$ or $[\nu](\pi)=0$. So

$$
([\mu] \cdot[\nu])(\pi)=0 \text {. }
$$

By Theorem 2.1, any constituent $[\lambda]$ of $[\mu] \cdot[\nu]$ satisfies

$$
\lambda_{1} \leq|\mu \cap \nu| \text { and } \lambda_{1}^{\prime} \leq\left|\mu \cap \nu^{\prime}\right|
$$

where $\lambda=\left(\lambda_{1}, \ldots\right), \lambda^{\prime}=\left(\lambda_{1}^{\prime}, \ldots\right)$. So the maximal possible hook length in $\lambda$ is $\ell$. Moreover, $\lambda$ contains a hook of length $\ell$ if and only if $\lambda_{1}=|\mu \cap \nu|$ and $\lambda_{1}^{\prime}=\left|\mu \cap \nu^{\prime}\right|$, in which case this is the $(1,1)$-hook whose leg length is $\left|\mu \cap \nu^{\prime}\right|-1$. In this case, using the Murnaghan-Nakayama Rule again, we get

$$
[\lambda](\pi)=(-1)^{\left|\mu \cap \nu^{\prime}\right|-1}\left[\lambda \backslash H_{11}\right](1) \neq 0
$$

where $\lambda \backslash H_{11}$ is the partition obtained from $\lambda$ by removing the $(1,1)$ hook $H_{11}$. Hence for every constituent $[\lambda]$ of $[\mu] \cdot[\nu]$ containing an $\ell$-hook we get a contribution on $\pi$ of the same sign, and so no cancellation can occur. But this contradicts Equation (2).

Theorem 3.4. Let $\mu, \nu$ be partitions of $n$, both different from $(n)$ and $\left(1^{n}\right)$. Then $[\mu] \cdot[\nu]$ is not homogenous. 
Proof. By Theorem 2.1, $[\mu] \cdot[\nu]$ has a constituent $[\lambda]$ with $\lambda_{1}=|\mu \cap \nu|$ and a constituent $[\kappa]$ with $\kappa_{1}^{\prime}=\left|\mu \cap \nu^{\prime}\right|$. If $\lambda=\kappa$, then $h_{11}^{\lambda}=|\mu \cap \nu|+\left|\mu \cap \nu^{\prime}\right|-1$, which is impossible by Theorem 3.3.

Corollary 3.5. A product $[\mu] \cdot[\nu]$ is irreducible if and only if at least one of the two characters $[\mu],[\nu]$ is of degree 1 .

\section{Kronecker products of $S_{n}$-representations with few components.}

The main result of this section is a description of the products of $S_{n^{-}}$ representations with two homogeneous components. First we need to know the product of any character with the character $[n-1,1]$ :

Lemma 4.1. Let $n \geq 3$ and $\mu$ be a partition of $n$. Then

$$
[\mu] \cdot[n-1,1]=\sum_{A} \sum_{B}\left[\left(\mu_{A}\right)^{B}\right]-[\mu]
$$

where the first sum is over all removable nodes $A$ for $\mu$, and the second sum runs over all addable nodes $B$ for $\mu_{A}$.

Proof. This follows from the isomorphisms $M^{(n-1,1)} \cong S^{(n-1,1)} \oplus S^{(n)}$ and $S^{\mu} \otimes M^{(n-1,1)} \cong\left(S^{\mu} \downarrow_{S_{n-1}}\right) \uparrow^{S_{n}}$.

Corollary 4.2. Let $n \geq 3$ and $\mu$ be a partition of $n$. Then:

(i) $[\mu] \cdot[n-1,1]$ has exactly one homogeneous component if and only if $\mu$ is $(n)$ or $\left(1^{n}\right)$.

(ii) $[\mu] \cdot[n-1,1]$ has exactly two homogeneous components if and only if $\mu$ is a rectangle $\left(a^{b}\right)$ for some $a, b>1$. In this case we have

$$
\left[a^{b}\right] \cdot[n-1,1]=\left[a+1, a^{b-2}, a-1\right]+\left[a^{b-1}, a-1,1\right] .
$$

(iii) $[\mu] \cdot[n-1,1]$ has exactly three homogeneous components if and only if $n=3$ and $\mu=(2,1)$. In this case we have

$$
[2,1] \cdot[2,1]=[3]+[2,1]+\left[1^{3}\right] .
$$

(iv) $[\mu] \cdot[n-1,1]$ has exactly four homogeneous components if and only if one of the following happens:

(a) $n \geq 4$ and $\mu=(n-1,1)$ or $\left(2,1^{n-2}\right)$;

(b) $\mu=(k+1, k)$ or $\left(2^{k}, 1\right)$ for $k \geq 2$.

We then have:

$$
\begin{aligned}
{[n-1,1] \cdot[n-1,1]=} & {[n]+[n-1,1]+[n-2,2]+\left[n-2,1^{2}\right], } \\
{[k+1, k] \cdot[2 k, 1]=} & {[k+2, k-1]+[k+1, k] } \\
& +[k+1, k-1,1]+\left[k^{2}, 1\right],
\end{aligned}
$$

and the remaining products are obtained by conjugation. 
Proof. The "if" parts and the decompositions of the products follow from Lemma 4.1.

We now prove the "only if" directions. We are going to use Lemma 4.1 again. First, observe that $[\mu]$ appears as a constituent in the product $[\mu] \otimes$ $[n-1,1]$ unless $\mu$ is a rectangle. Also note that $\left(\mu_{A}\right)^{B}=\left(\mu_{A^{\prime}}\right)^{B^{\prime}}$ for two different pairs $(A, B),\left(A^{\prime}, B^{\prime}\right)$ if and only if $A=B$ and $A^{\prime}=B^{\prime}$, in which case $\left(\mu_{A}\right)^{B}=\left(\mu_{A^{\prime}}\right)^{B^{\prime}}=\mu$.

A partition with $r$ removable nodes has exactly $r+1$ addable nodes. So if $\mu$ has at least 2 removable nodes, say $A_{1}$ and $A_{2}$, then $\mu_{A_{1}}$ and $\mu_{A_{2}}$ both have at least 2 addable nodes, which gives 4 composition factors in the product with the only common constituent $[\mu]$. This proves the "only if" part of (i) and (ii). If $\mu$ has at least 3 removable nodes, then a similar argument shows that $[\mu] \cdot[n-1,1]$ has at least 5 non-isomorphic constituents. So we may assume that $\mu$ has exactly two removable nodes: $A_{1}$ and $A_{2}$. For $[\mu] \cdot[n-1,1]$ to have exactly 3 components, both $\mu_{A_{1}}$ and $\mu_{A_{2}}$ should have only one removable node. This is only possible if $n=3$ and $\mu=(2,1)$. Finally, for $[\mu] \cdot[n-1,1]$ to have exactly 4 components, one of $\mu_{A_{1}}$ and $\mu_{A_{2}}$ should have only one removable node and the other one should have two. This occurs exactly if $\mu$ or $\mu^{\prime}$ is $(n-1,1), n \geq 4$, or $(k+1, k), k \geq 2$.

Lemma 4.3. Let $\lambda$ be a partition of $n$. Then the square $[\lambda]^{2}$ has at most 4 homogeneous components if and only if one of the following holds:

(i) $\lambda=(n)$ or $\left(1^{n}\right)$, when $[\lambda]^{2}=[n]$;

(ii) $n \geq 4, \lambda=(n-1,1)$ or $\left(2,1^{n-2}\right)$, when $[\lambda]^{2}=[n]+[n-1,1]+[n-$ $2,2]+\left[n-2,1^{2}\right]$

(iii) $n=3, \lambda=(2,1)$, when $[\lambda]^{2}=[3]+[2,1]+\left[1^{3}\right]$;

(iv) $n=4, \lambda=\left(2^{2}\right)$, when $[\lambda]^{2}=[4]+\left[2^{2}\right]+\left[1^{4}\right]$;

(v) $n=6, \lambda=\left(3^{2}\right)$ or $\left(2^{3}\right)$, when $[\lambda]^{2}=[6]+[4,2]+\left[3,1^{3}\right]+\left[2^{3}\right]$.

Proof. The "if" part follows from Corollary 4.2 and [4, Tables I.I].

In the other direction, let $[\lambda]^{2}$ have at most 4 homogeneous components. We may assume that $\lambda$ is not one of $(n),\left(1^{n}\right),(n-1,1),\left(2,1^{n-2}\right)$, and that $n>8$ since for $n \leq 8$ the results hold by [4, Tables I.I].

Clearly $[\lambda]^{2}$ always contains $[n]$. Furthermore, by [10, Lemmas 1-3] and $[\mathbf{1 2}, 4.3]$ or by $[\mathbf{1 1}, 6.3],[\lambda]^{2}$ contains $[n-2,2]$, and unless $\lambda$ is a rectangle, it also contains $[n-1,1],\left[n-2,1^{2}\right]$ and $[n-3,3]$. So we only have to deal with the case where $\lambda=\left(a^{b}\right)$ is a rectangle. We already know that $[\lambda]^{2}$ has the constituents $[n]$ and $[n-2,2]$. If $b>2$, then $[\lambda]^{2}$ also has the constituent $[n-3,3]$ by $[\mathbf{1 0}$, Lemma 3] or $[\mathbf{1 1}, 6.3]$. If $n>12$, then also $[n-4,4]$ occurs, see [10, Lemma 4]. Furthermore, by $[\mathbf{1 1}, 6.3],\left[n-3,1^{3}\right]$ appears as a constituent. Hence we can restrict ourselves to the cases $\lambda=(k, k)$ or $\lambda=\left(4^{3}\right)$.

Suppose $\lambda=(k, k)(k \geq 5)$. By Corollary 2.4, the components $[\mu]$ of $[k, k]^{2}$ with $\mu_{1}^{\prime}=4=\left|\lambda \cap \lambda^{\prime}\right|$ are of the form $\left(\rho_{1}+1, \rho_{2}+1, \rho_{3}+1, \rho_{4}+1\right)$, 
where $\left[\left(\rho_{1}, \rho_{2}, \rho_{3}, \rho_{4}\right)\right]$, is a constituent of $[k-2, k-2]^{2}$. By what we have already proved, there are at least 3 such constituents. Thus $[k, k]^{2}$ has at least 5 components.

Now, let $\lambda=\left(4^{3}\right)$. We already know that $[\lambda]^{2}$ contains $[12],[10,2],[9,3]$ and $\left[9,1^{3}\right]$. But it also contains some $[\mu]$ with $\mu_{1}^{\prime}=9=\left|\lambda \cap \lambda^{\prime}\right|$, thanks to Theorem 2.1. Alternatively, one may calculate $\left[4^{3}\right]^{2}$ on a computer and find 52 (!) homogeneous components.

Lemma 4.4. Let $\mu, \gamma$ be partitions, $\gamma \subset \mu$. Set $I=\left\{i \mid \gamma_{i}<\mu_{i}\right\}$. Then the following assertions are equivalent:

(i) $[\mu / \gamma]$ is homogeneous;

(ii) $[\mu / \gamma]$ is irreducible;

(iii) $I=\{j, j+1, \ldots, k\}$ for some $j \leq k$, and one of the following holds:

(a) $\gamma_{j}=\gamma_{j+1}=\cdots=\gamma_{k}$;

(b) $\mu_{j}=\mu_{j+1}=\cdots=\mu_{k}$.

Moreover, in this case $[\mu / \gamma]=[\alpha]$, where $\alpha$ is the partition with the parts $\mu_{i}-\gamma_{i}, i \in I$, sorted in the weakly decreasing order.

Proof. This follows from the Littlewood-Richardson Rule.

Remark. The situations described in (iii)(a) and (iii)(b) above correspond respectively to the pictures
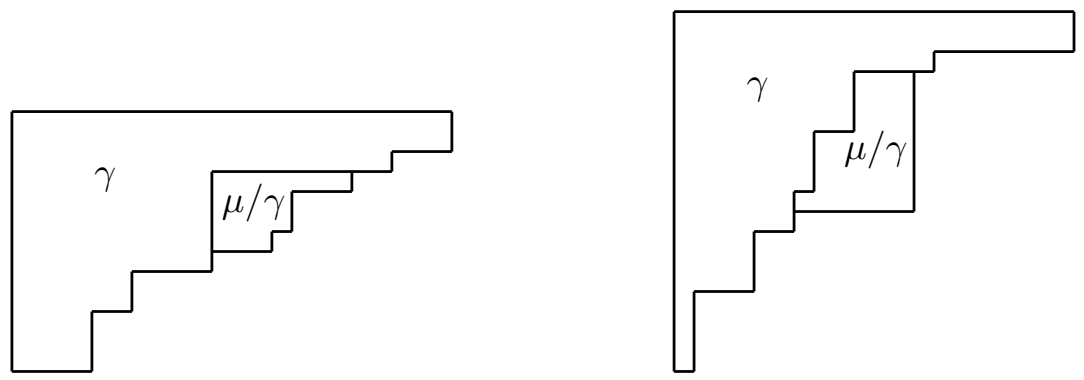

Lemma 4.5. In the notation of Lemma 4.4 (and under the same assumptions), let $A$ be a removable node of $\gamma$.

(1) If $A$ is disconnected from $\mu / \gamma$ then

$$
\left[\mu / \gamma_{A}\right]=\sum_{B}\left[\alpha^{B}\right]
$$

where $B$ runs over the addable nodes of $\alpha$.

(2) Let $A$ be connected with $\mu / \gamma$.

In the case (iii)(a) we have

$$
\left[\mu / \gamma_{A}\right]=\sum_{B \neq B_{0}}\left[\alpha^{B}\right]
$$


where $B$ runs over the addable nodes of $\alpha$, except for the bottom addable node $B_{0}$.

In the case (iii)(b) we have

$$
\left[\mu / \gamma_{A}\right]=\left[\alpha^{B}\right]
$$

where $B$ is an addable node of $\alpha$.

Proof. Again, this follows by the Littlewood-Richardson Rule.

The following two lemmas will be used in the proof of the main theorem of this section.

Lemma 4.6. Let $\mu \neq \nu$ be partitions of $n$, both different from $(n),\left(1^{n}\right)$, $(n-1,1)$ and $\left(2,1^{n-2}\right)$. Put $\gamma=\mu \cap \nu, m=|\gamma|$. Assume that $\nu / \gamma$ is a row and that $[\mu / \gamma]$ is an irreducible character $\left[\alpha_{1}, \alpha_{2}, \ldots\right]$. Then $\left[m, \alpha_{1}, \alpha_{2}, \ldots\right]$ appears in $[\mu] \cdot[\nu]$. Moreover if an $S_{n-m+1}$-character $\left[\theta_{1}, \theta_{2}, \ldots\right]$ appears in

$$
\sum_{A \text { removable for } \gamma}\left[\mu / \gamma_{A}\right] \cdot\left[\nu / \gamma_{A}\right]-\sum_{B \text { addable for } \alpha}\left[\alpha^{B}\right]
$$

with a positive coefficient then $\left[m-1, \theta_{1}, \theta_{2}, \ldots\right]$ appears in $[\mu] \cdot[\nu]$.

Proof. We have $[\nu / \gamma]=[n-m]$. So Theorem 2.1 and Corollary 2.3 yield:

$$
\left\langle[\mu] \cdot[\nu],\left[m, \alpha_{1}, \alpha_{2}, \ldots\right]\right\rangle=1,
$$

and

$$
\text { if } \lambda \neq\left(m, \alpha_{1}, \alpha_{2}, \ldots\right) \text { and }\langle[\mu] \cdot[\nu],[\lambda]\rangle \neq 0 \text { then } \lambda_{1}<m \text {. }
$$

If $\lambda$ is a partition of $n$ with $\lambda_{1}=m-1$, then in the notation of Theorem 2.2, we may write

$$
\begin{aligned}
\left\{\eta \in Y(\lambda) \mid \eta \neq \lambda, \eta_{1} \leq m\right\} & \\
& =\left\{\left(m, \lambda_{2}, \ldots, \lambda_{i-1}, \lambda_{i}-1, \lambda_{i+1}, \ldots\right) \mid i \geq 1, \lambda_{i}>\lambda_{i+1}\right\}
\end{aligned}
$$

So (4) and (5) imply $\sum_{\substack{\eta \in Y(\lambda) \\ \eta \neq \lambda \\ \eta_{1} \leq m}} d(\mu, \nu ; \eta)=\varepsilon$, where

$$
\varepsilon= \begin{cases}1 & \text { if } \hat{\lambda}=\alpha^{B} \text { for some addable node } B \text { of } \alpha \\ 0 & \text { otherwise. }\end{cases}
$$

Now, by Theorem 2.2, for a partition $\lambda$ of $n$ with $\lambda_{1}=m-1$ we have

$$
\langle[\mu] \cdot[\nu],[\lambda]\rangle=\sum_{A}\left\langle\left[\mu / \gamma_{A}\right] \cdot\left[\nu / \gamma_{A}\right],[\hat{\lambda}]\right\rangle-\varepsilon
$$

where the sum is over all removable nodes $A$ of $\gamma$.

Let $[\theta]$ be a constituent of $\left[\mu / \gamma_{A}\right] \cdot\left[\nu / \gamma_{A}\right]$. Then $[\theta]$ is a constituent of $[\beta] \cdot[\delta]$ with $[\beta]$ a constituent of $\left[\mu / \gamma_{A}\right]$ and $[\delta]$ a constituent of $\left[\nu / \gamma_{A}\right]$. It 
follows from the definition of skew characters that $\beta \subseteq \mu, \delta \subseteq \nu$. Hence $\beta \cap \delta \subseteq \mu \cap \nu=\gamma$. In view of Theorem 2.1, this implies

$$
\theta_{1} \leq|\beta \cap \delta| \leq|\mu \cap \nu|=m .
$$

If $\theta_{1}=m$, then $\beta \cap \delta=\gamma$, therefore $\beta \supseteq \gamma$ and $\delta \supseteq \gamma$. However, $\nu / \gamma_{A}$ is a union of a row and a node, so either $\delta=(n-m+1)$ or $\delta=(n-m, 1)$. If $\delta=(n-m+1)$, then $\mu \cap \nu \subseteq \delta$ implies $\mu \cap \nu=(m)$. But then either $\mu$ or $\nu$ is $(n)$, which contradicts the assumptions of the lemma. If $\delta=(n-m, 1)$, then we conclude similarly that $\mu \cap \nu=(m-1,1)$. Since neither $\mu$ nor $\nu$ is equal to $(n-1,1)$ or its conjugate and $\mu / \gamma$ should be connected by Lemma 4.4 , then the only possibilities are: $\nu=(m-1, n-m+1), \mu=\left(m-1,1^{n-m+1}\right)$ or $\nu=\left(n-2,1^{2}\right), \mu=(n-2,2)$ (in the latter case $\left.n-m=1\right)$. In both cases $m-1 \geq n-m+1$, so $\theta_{1} \leq m-1$ since $\theta$ is a partition of $n-m+1$. This contradiction shows that we may assume that $\theta_{1} \leq m-1$ for any $[\theta]$ appearing in $\left[\mu / \gamma_{A}\right] \cdot\left[\nu / \gamma_{A}\right]$.

This, together with $(7)$, shows that any $S_{n-m+1}$-character $\left[\theta_{1}, \theta_{2}, \ldots\right]$ appearing in (3) gives rise to the character $\left[m-1, \theta_{1}, \theta_{2}, \ldots\right]$ appearing in $[\mu] \cdot[\nu]$.

Lemma 4.7. Let $\mu \neq \nu$ be partitions of $n$, both different from $(n),\left(1^{n}\right),(n-$ $1,1)$, and $\left(2,1^{n-2}\right)$. Put $\gamma=\mu \cap \nu$. Assume that $\nu / \gamma$ is a row, $[\mu / \gamma]$ is irreducible, and $[\mu] \cdot[\nu]$ has 2 homogeneous components.

If there exists a removable node $A_{0}$ of $\gamma$, disconnected from $\nu / \gamma$, then the following condition holds:

$\left.{ }^{*}\right)\left[\mu / \gamma_{A_{0}}\right]$ is 1-dimensional, $\mu / \gamma$ is connected with all removable nodes of $\gamma, \nu / \gamma$ is connected with all removable nodes of $\gamma$ except $A_{0}$.

Proof. Let $A_{0}$ be a removable node of $\gamma$ disconnected from $\nu / \gamma$, and put $m=|\gamma|$. Since $\mu \neq \nu$, we have $n-m>0$. Let $\alpha$ be the partition of $n-m$ defined by $[\mu / \gamma]=[\alpha]$. Note that $[\nu / \gamma]=[n-m]$.

By Lemma 4.6, it suffices to show that the expression (3) contains at least two distinct irreducible characters unless the conditions $\left(^{*}\right)$ hold.

Since $A_{0}$ is disconnected from $\nu / \gamma$, we have by Lemma 4.5(1):

$$
\left[\nu / \gamma_{A_{0}}\right]=[n-m+1]+[n-m, 1] .
$$

In view of Lemmas 4.4 and 4.5, we have three cases to consider: (a) When $A_{0}$ is disconnected from $\mu / \gamma$; (b) when $A_{0}$ is connected with $\mu / \gamma$ and we are in the case (iii)(a) of Lemma 4.4; (c) when $A_{0}$ is connected with $\mu / \gamma$ and we are in the case (iii)(b) of Lemma 4.4 (the cases (b) and (c) overlap when $\mu / \gamma$ is a rectangle).

(a) In this case $A_{0}$ is disconnected from $\mu / \gamma$. Then, by Lemma 4.5(1), we get

$$
\left[\mu / \gamma_{A_{0}}\right]=\sum_{B}\left[\alpha^{B}\right]
$$


where the sum runs over all addable nodes $B$ of $\alpha$. So (3) contains

$$
\begin{aligned}
& ([n-m+1]+[n-m, 1]) \cdot\left(\sum_{B}\left[\alpha^{B}\right]\right)-\sum_{B}\left[\alpha^{B}\right] \\
& =[n-m, 1] \cdot\left(\sum_{B}\left[\alpha^{B}\right]\right) .
\end{aligned}
$$

If there is a non-linear character among the $\left[\alpha^{B}\right]$, we are done by Corollary 4.2(i). Otherwise $\alpha=(1)$, but even in this case the expression above contains two different characters: [2] and [ $\left.1^{2}\right]$. This completes the case (a).

In particular, we now may assume that every removable node $A$ of $\gamma$ disconnected from $\nu / \gamma$ is connected with $\mu / \gamma$.

Note that $\left[\mu / \gamma_{A_{0}}\right]$ contains $\left[\alpha^{B_{1}}\right]$ for some addable node $B_{1}$, see Lemma 4.5. So, in view of (8) and Lemma 4.1, $\left[\mu / \gamma_{A_{0}}\right] \cdot\left[\nu / \gamma_{A_{0}}\right]$ contains $\sum_{B}\left[\alpha^{B}\right]$. Hence any removable node $A_{1} \neq A_{0}$ of $\gamma$ yields a positive contribution of $\left[\mu / \gamma_{A_{1}}\right] \cdot\left[\nu / \gamma_{A_{1}}\right]$ to the expression (3). If $A_{1}$ is disconnected from $\nu / \gamma$ then $\left[\nu / \gamma_{A_{1}}\right]=[n-m, 1]+[n-m+1]$, and the product $\left[\mu / \gamma_{A_{1}}\right] \cdot\left[\nu / \gamma_{A_{1}}\right]$ is not homogeneous. If $A_{1}$ is connected with $\nu / \gamma$ but disconnected from $\mu / \gamma$ then, by Lemma $4.5,\left[\mu / \gamma_{A_{1}}\right]$ is not irreducible and $\left[\nu / \gamma_{A_{1}}\right]$ is $[n-m, 1]$ or $[n-m+1]$. So the product $\left[\mu / \gamma_{A_{1}}\right] \cdot\left[\nu / \gamma_{A_{1}}\right]$ is not homogeneous again, thanks to Lemmas 4.1 and 4.5. Thus we may always assume that:

(**) $\mu / \gamma$ is connected with all removable nodes of $\gamma$, and $\nu / \gamma$ is connected with all removable nodes of $\gamma$ different from $A_{0}$.

(b) In this case Lemma 4.5 yields

$$
\left[\mu / \gamma_{A_{0}}\right]=\sum_{B \neq B_{0}}\left[\alpha^{B}\right]
$$

where the sum runs over all addable nodes $B$ of $\alpha$ except for the bottom one $B_{0}$. Consider the constituent $\left[\mu / \gamma_{A_{0}}\right] \cdot\left[\nu / \gamma_{A_{0}}\right]-\sum_{B}\left[\alpha^{B}\right]$ of (3). By (8), it is equal to

$$
\begin{aligned}
& ([n-m+1]+[n-m, 1]) \cdot\left(\sum_{B \neq B_{0}}\left[\alpha^{B}\right]\right)-\sum_{B}\left[\alpha^{B}\right] \\
& =[n-m, 1] \cdot\left(\sum_{B \neq B_{0}}\left[\alpha^{B}\right]\right)-\left[\alpha^{B_{0}}\right] .
\end{aligned}
$$

Since $\alpha \neq \emptyset$, it has at least 2 addable nodes. Let $B_{1}$ be an addable node of $\alpha$, different from $B_{0}$, and let $r$ be the number of removable nodes of $\alpha^{B_{1}}$. 
Then, using Lemma 4.1, we can rewrite (9) as follows:

$$
\begin{aligned}
& {[n-m, 1] \cdot\left(\left[\alpha^{B_{1}}\right]+\sum_{B \neq B_{0}, B_{1}}\left[\alpha^{B}\right]\right)-\left[\alpha^{B_{0}}\right]} \\
& =(r-1)\left[\alpha^{B_{1}}\right]+\left[\alpha^{B_{0}}\right]+\sum_{B \neq B_{0}, B_{1}}\left[\alpha^{B}\right]+\sum_{C, D}\left[\left(\alpha^{B_{1}}\right)_{C}{ }^{D}\right] \\
& \quad+[n-m, 1] \cdot\left(\sum_{B \neq B_{0}, B_{1}}\left[\alpha^{B}\right]\right)-\left[\alpha^{B_{0}}\right] \\
& =(r-1)\left[\alpha^{B_{1}}\right]+([n-m+1]+[n-m, 1]) \cdot\left(\sum_{B \neq B_{0}, B_{1}}\left[\alpha^{B}\right]\right) \\
& \quad+\sum_{C, D}\left[\left(\alpha^{B_{1}}\right)_{C}^{D}\right]
\end{aligned}
$$

where the sum $\sum_{C, D}$ is over all removable nodes $C$ of $\alpha^{B_{1}}$, different from $B_{1}$, and over all addable nodes $D$ of $\left(\alpha^{B_{1}}\right)_{C}$, different from $C$.

If $\alpha$ is not a rectangle, then $\sum_{B \neq B_{0}, B_{1}}$ is non-empty, so our expression involves at least two different irreducible characters. Let $\alpha$ be a rectangle. If $[\alpha]$ is not of degree 1 , then $\alpha^{B_{1}}$ is not a rectangle, so $r>1$, and thus our expression involves $\left[\alpha^{B_{1}}\right]$. Moreover, $\alpha^{B_{1}}$ has a removable node $C \neq B_{1}$, so for an addable node $D \neq C$ of $\left(\alpha^{B_{1}}\right)_{C}$ we get the contribution $\left[\left(\alpha^{B_{1}}\right)_{C}{ }^{D}\right] \neq$ $\left[\alpha^{B_{1}}\right]$. Finally, let $[\alpha]=[\mu / \gamma]$ be of degree 1 . If $\left[\alpha^{B_{1}}\right]$ is not of degree 1 , then it is $\left[2,1^{(n-m-1)}\right]$. So for $n-m \geq 2$, we have $r=2$, and so $\left[2,1^{n-2}\right]$ and $\left[3,1^{n-3}\right]$ appear in our expression. However, if $n-m=1$, then $\left[\mu / \gamma_{A_{0}}\right]$ is of degree 1 . So, in view of $(* *)$, all the conditions in $\left(^{*}\right)$ hold.

(c) In this case by Lemma 4.5 we have

$$
\left[\mu / \gamma_{A_{0}}\right]=\left[\alpha^{B_{1}}\right]
$$

for some addable node $B_{1}$ of $\alpha$. Then the constituent $\left[\mu / \gamma_{A_{0}}\right] \cdot\left[\nu / \gamma_{A_{0}}\right]-$ $\sum_{B}\left[\alpha^{B}\right]$ of $(3)$ is

$$
\begin{aligned}
& ([n-m+1]+[n-m, 1]) \cdot\left[\alpha^{B_{1}}\right]-\sum_{B}\left[\alpha^{B}\right] \\
& =\sum_{C, D}\left[\left(\alpha^{B_{1}}\right)_{C}{ }^{D}\right]-\sum_{B}\left[\alpha^{B}\right] \\
& =\sum_{B}\left[\alpha^{B}\right]+\sum_{C, D ; C \neq B_{1}}\left[\left(\alpha^{B_{1}}\right)_{C}{ }^{D}\right]-\sum_{B}\left[\alpha^{B}\right]
\end{aligned}
$$




$$
=\sum_{C, D ; C \neq B_{1}}\left[\left(\alpha^{B_{1}}\right)_{C}^{D}\right] .
$$

In the last sum $C$ runs through the removable nodes of $\alpha^{B_{1}}$, different from $B_{1}$, and $D$ runs through the addable nodes of $\left(\alpha^{B_{1}}\right)_{C}$. So (10) has at least two different irreducible constituents, unless it is empty. Hence we may assume that $\alpha^{B_{1}}$ is a rectangle. If $[\alpha]$ is of degree 1 then $\left[\alpha^{B_{1}}\right]=\left[\mu / \gamma_{A_{0}}\right]$ is also of degree 1 , and, in view of $\left({ }^{* *}\right)$, we are in the exceptional case $\left(^{*}\right)$. So we may assume that $\alpha=\left(a^{b-1}, a-1\right)$ for some $a>1, b>1$, and $\alpha^{B_{1}}=\left(a^{b}\right)$. This together with Lemma 4.4 implies that $\gamma$ has a removable node $A_{1}$, different from $A_{0}$. We know that it must be connected with $\nu / \gamma$ and $\mu / \gamma$, thanks to $(* *)$. If there was a third removable node of $\gamma, A_{2}$ say, then again by $\left({ }^{* *}\right)$, both $A_{1}$ and $A_{2}$ would be connected with both $\nu / \gamma$ and $\mu / \gamma$. But this is impossible since $\gamma=\mu \cap \nu$. So we may assume that $\gamma$ has exactly two removable nodes. Now, by Lemma 4.5(2), we have $\left[\mu / \gamma_{A_{1}}\right]=\left[\alpha^{B_{2}}\right]$ with $B_{2}$ the top or the bottom, but not the middle, addable node of $\alpha$, and $\left[\nu / \gamma_{A_{1}}\right]$ is either $[n-m, 1]$ or $[n-m+1]$. The corresponding pictures are:
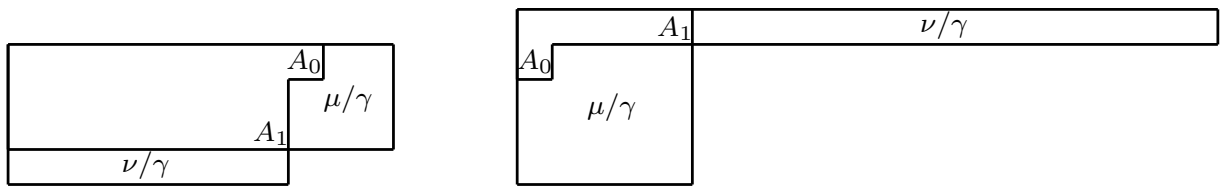

In the first case, $\left[\alpha^{B_{2}}\right] \cdot[n-m, 1]$ contributes at least two constituents by Theorem 3.4. In the second case $\nu=(n-1,1)$.

Theorem 4.8. Let $\mu, \nu$ be partitions of $n$. Then $[\mu] \cdot[\nu]$ has exactly two homogenous components if and only if one of the partitions $\mu, \nu$ is a rectangle $\left(a^{b}\right)$ with $a, b>1$, and the other is $(n-1,1)$ or $\left(2,1^{n-2}\right)$. In these cases we have:

$$
\begin{aligned}
{[n-1,1] \cdot\left[a^{b}\right] } & =\left[a+1, a^{b-2}, a-1\right]+\left[a^{b-1}, a-1,1\right], \\
{\left[2,1^{n-2}\right] \cdot\left[a^{b}\right] } & =\left[b+1, b^{a-2}, b-1\right]+\left[b^{a-1}, b-1,1\right] .
\end{aligned}
$$

Proof. The "if" part is proved in Corollary 4.2 (note that $S^{\left(2,1^{n-2}\right)} \cong$ $S^{(n-1,1)} \otimes$ sign). To prove the "only if" part, assume that

$$
[\mu] \cdot[\nu]=x[\kappa]+y[\lambda] \text { for some } x, y \in \mathbb{N},
$$

with $\kappa>\lambda$ in the lexicographic order. Clearly, $\mu, \nu \notin\left\{(n),\left(1^{n}\right)\right\}$. If $\mu$ or $\nu$ is $(n-1,1)$ or $\left(2,1^{n-2}\right)$ the result follows from Corollary 4.2. Assume $\mu, \nu \notin\left\{(n-1,1),\left(2,1^{n-2}\right)\right\}$. By Theorems 2.1 and 3.3, we have

$$
\kappa_{1}=|\mu \cap \nu|, \quad \lambda_{1}^{\prime}=\left|\mu \cap \nu^{\prime}\right| \quad \text { and } \quad \lambda_{1}<|\mu \cap \nu|=\kappa_{1} .
$$


By Lemma 4.3, $\mu \neq \nu$, and hence $\kappa_{1}<n$. Put $\gamma=\mu \cap \nu, m=|\gamma|$. By Corollary 2.3, we must have

$$
[\mu / \gamma] \cdot[\nu / \gamma]=x[\hat{\kappa}]
$$

where $\hat{\kappa}=\left(\kappa_{2}, \kappa_{3}, \ldots\right)$. So, in view of Theorem 3.4, one of the following happens:

(i) $x=1$ and one of the characters $[\mu / \gamma],[\nu / \gamma]$ is of degree 1 , while the other is irreducible;

(ii) one of the characters $[\mu / \gamma],[\nu / \gamma]$ is equal to $[\hat{\kappa}]$, the other is of the form $z[n-m]+w\left[1^{n-m}\right]$ with some $z, w \in \mathbb{N}$, and $\hat{\kappa}=\hat{\kappa}^{\prime}$. By the LittlewoodRichardson rule, a skew character contains both $[n-m]$ and $\left[1^{n-m}\right]$ only if its diagram is a set of disconnected nodes. So we must have $n-m=2$, since otherwise such a skew character has more than 2 constituents. But there is no symmetric partition of 2 , i.e. $\hat{\kappa} \neq \hat{\kappa}^{\prime}$. This contradiction allows us to assume that we are in the case (i).

Without loss of generality, suppose that $[\nu / \gamma]$ is of degree 1 and $[\mu / \gamma]=$ $[\alpha]$ is irreducible. Then the shape of $\nu / \gamma$ is a row or a column. Passing, if necessary, from $\mu, \nu$ to $\mu^{\prime}, \nu^{\prime}$, we may assume that $\nu / \gamma$ is a row. Now, by Lemma 4.7 we may assume that one of the following holds:

(a) $\nu / \gamma$ is connected with every removable node of $\gamma$.

(b) There exists a removable node $A_{0}$ of $\gamma$ disconnected from $\nu / \gamma,[\mu / \gamma]$ and $\left[\mu / \gamma_{A_{0}}\right]$ are of degree $1, \mu / \gamma$ is connected with every removable node of $\gamma$, and $\nu / \gamma$ is connected with every removable node of $\gamma$ different from $A_{0}$.

Case (a). In this case $\nu$ must be a rectangle, and $\gamma$ must have a removable node $A_{0}$ such that $\left[\nu / \gamma_{A_{0}}\right]=(n-m, 1)$ for otherwise $\nu=(n)$.

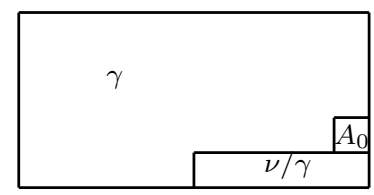

Let us first assume that $\mu / \gamma$ is disconnected from $A_{0}$. Then, in view of Lemmas 4.5(1) and 4.1, the expression (3) contains

$$
\begin{aligned}
& {\left[\nu / \gamma_{A_{0}}\right] \cdot\left[\mu / \gamma_{A_{0}}\right]-\sum_{B}\left[\alpha^{B}\right]} \\
& =[n-m, 1] \cdot\left(\sum_{B}\left[\alpha^{B}\right]\right)-\sum_{B}\left[\alpha^{B}\right] \\
& =\sum_{B} \sum_{C} \sum_{D}\left[\left(\alpha^{B}\right)_{C}{ }^{D}\right]-2 \sum_{B}\left[\alpha^{B}\right]
\end{aligned}
$$




$$
=\sum_{B}\left(r_{B}-2\right)\left[\alpha^{B}\right]+\sum_{B} \sum_{C} \sum_{D \neq C}\left[\left(\alpha^{B}\right)_{C}{ }^{D}\right]
$$

where $B$ runs over the addable nodes of $\alpha, C$ runs over the removable nodes of $\alpha^{B}$ (for the respective node $B$ ), $D$ runs over the addable nodes of $\left(\alpha^{B}\right)_{C}$ and $r_{B}$ denotes the number of removable nodes of $\alpha^{B}$.

If $\alpha$ has at least 3 addable nodes, say $B_{0}, B_{1}, B_{2}$, then we have the following contribution to the expression above:

$$
\begin{aligned}
& \left(r_{B_{0}}-2\right)\left[\alpha^{B_{0}}\right]+\left[\alpha^{B_{1}}\right]+\left[\alpha^{B_{2}}\right]+\left(r_{B_{1}}-2\right)\left[\alpha^{B_{1}}\right]+\left[\alpha^{B_{0}}\right]+\left[\alpha^{B_{2}}\right] \\
& \quad+\left(r_{B_{2}}-2\right)\left[\alpha^{B_{2}}\right]+\left[\alpha^{B_{0}}\right]+\left[\alpha^{B_{1}}\right] \\
& =r_{B_{0}}\left[\alpha^{B_{0}}\right]+r_{B_{1}}\left[\alpha^{B_{1}}\right]+r_{B_{2}}\left[\alpha^{B_{2}}\right] .
\end{aligned}
$$

By Lemma 4.6, this yields 3 irreducible components in $[\mu] \cdot[\nu]$.

So $\alpha$ has exactly two addable nodes, say $B_{0}, B_{1}$, i.e. $\alpha$ is a rectangle. Then we have the following contribution to the expression (11):

$$
\left(r_{B_{0}}-2\right)\left[\alpha^{B_{0}}\right]+\left[\alpha^{B_{1}}\right]+\left(r_{B_{1}}-2\right)\left[\alpha^{B_{1}}\right]+\left[\alpha^{B_{0}}\right] .
$$

If $\alpha$ is not a row or a column then both $r_{B_{0}}, r_{B_{1}}$ are at least 2 , and in view of Lemma 4.6, we get two irreducible constituents for $[\mu] \cdot[\nu]$, both different from $[\kappa]$. Let $\alpha$ be a row or a column. Assume that $\alpha$ is a row, the column case being similar. Then (11) equals $[n-m, 1]+[n-m-1,2]+\left[n-m-1,1^{2}\right]$ if $n-m>2$, and $[2,1]+\left[1^{3}\right]$ if $n-m=2$. By Lemma 4.6, this yields at least two constituents in $[\mu] \cdot[\nu]$ different from $[\kappa]$. Finally, let $n-m=1$. Then (11) equals 0 . Note that $\gamma$ must have a removable node $A_{1} \neq A_{0}$, since otherwise $\nu=\left(1^{n}\right)$. If $\mu / \gamma$ is disconnected from $A_{1}$, then

$$
\left[\mu / \gamma_{A_{1}}\right] \cdot\left[\nu / \gamma_{A_{1}}\right]=[2]+\left[1^{2}\right],
$$

and we are done by Lemma 4.6. If $\mu / \gamma$ is connected with $A_{1}$, then $\mu=$ $\left(2^{k}, 1^{2}\right), \nu=\left(2^{k+1}\right)$ (and $k>1$ since $\mu$ is not of the form $\left(2,1^{n-2}\right)$ ). Then the expression (3) equals $\left[1^{2}\right]$. So, by Lemma $4.6,[n-1,1]$ and $\left[n-2,1^{2}\right]$ are constituents of $[\mu] \cdot[\nu]$. But $\left|\mu \cap \nu^{\prime}\right|=4$, so there also must be a constituent with 4 non-zero rows, thanks to Theorem 2.1.

This completes the consideration of the case where $[\mu / \gamma]$ is disconnected from $A_{0}$.

Let $\mu / \gamma$ be connected with $A_{0}$. Then, in view of Lemmas 4.4 and $4.5(2)$, we have

$$
\left[\mu / \gamma_{A_{0}}\right]=\sum_{B \neq B_{0}}\left[\alpha^{B}\right]
$$

where $B_{0}$ is the bottom addable node of $\alpha$. Let $B_{1}$ be the top addable node of $\alpha$. Then we get a contribution to (3) from the following expression:

$$
\left[\nu / \gamma_{A_{0}}\right] \cdot\left[\mu / \gamma_{A_{0}}\right]-\sum_{B}\left[\alpha^{B}\right]
$$




$$
\begin{aligned}
& =[n-m, 1] \cdot \sum_{B \neq B_{0}}\left[\alpha^{B}\right]-\sum_{B}\left[\alpha^{B}\right] \\
& =\sum_{B \neq B_{0}} \sum_{C} \sum_{D}\left[\left(\alpha^{B}\right)_{C}{ }^{D}\right]-\sum_{B \neq B_{0}}\left[\alpha^{B}\right]-\sum_{B}\left[\alpha^{B}\right] \\
& =\sum_{C \neq B_{1}} \sum_{D}\left[\left(\alpha^{B_{1}}\right)_{C}^{D}\right]+\sum_{B \neq B_{0}, B_{1}} \sum_{C} \sum_{D}\left[\left(\alpha^{B}\right)_{C}{ }^{D}\right]-\sum_{B \neq B_{0}}\left[\alpha^{B}\right]
\end{aligned}
$$

where $B$ runs through the addable nodes of $\alpha, C$ runs through the removable nodes of $\alpha^{B}$ (for the respective node $B$ ) and $D$ runs through the addable nodes of $\left(\alpha^{B}\right)_{C}$.

If $\alpha$ has a third addable node, say $B_{2}$, then $\alpha^{B_{1}}$ is not a rectangle, and hence there exists a node $C_{1} \neq B_{1}$ which is removable from $\alpha^{B_{1}}$. This shows that the first sum in (12) contains $\left[\alpha^{B_{1}}\right]$. Moreover, the second sum in (12) contains $\sum_{D}\left[\alpha^{D}\right]$, and so both $\left[\alpha^{B_{0}}\right]$ and $\left[\alpha^{B_{1}}\right]$ are constituents of (12). Now we can apply Lemma 4.6.

If $B_{0}$ and $B_{1}$ are the only addable nodes of $\alpha$, then $\alpha$ is a rectangle. Let $C_{1}$ be the corner node of $\alpha$.

If $\alpha$ is not a row, then $\alpha^{B_{1}}$ also has the removable node $C_{1}$. In this case, (12) is

$$
\sum_{D}\left[\left(\alpha^{B_{1}}\right)_{C_{1}}^{D}\right]-\left[\alpha^{B_{1}}\right]
$$

which gives at least two contributions, except in the case where $\alpha=\left(1^{2}\right)$ when (12) equals [3]. If $\gamma$ has a further removable node $A_{1}$, then this leads to a further contribution $[2,1]$ to $(3)$. But if $\gamma$ is a rectangle, then $\mu=\left(2^{3}\right)$ and $\nu=\left(3^{2}\right)$, and we can apply Lemma 4.3 .

If $\alpha$ is a row then $\gamma$ must have a removable node $A_{1} \neq A_{0}$, since otherwise $\mu=(n)$. Note that $(12)$ equals $-[n-m+1]$. Also $\left[\nu / \gamma_{A_{1}}\right] \cdot\left[\mu / \gamma_{A_{1}}\right]=[n-m+$ $1]+[n-m, 1]$. By Lemma 4.6, the product $[\mu] \cdot[\nu]$ contains $[m, n-m]$ and $[m-1, n-m, 1]$. Note that our assumptions yield $\mu=(k+n-m, k-n+m)$, $\nu=(k, k)$ with $k-n+m \geq 2$. But in this case $\left|\mu \cap \nu^{\prime}\right| \geq 4$. So Theorem 2.1 implies that $[\mu] \cdot[\nu]$ has a constituent with 4 rows.

Case (b). Since $[\mu]$ is not of degree 1 , the assumption $[\mu / \gamma]$ and $\left[\mu / \gamma_{A_{0}}\right]$ are of degree 1 implies that $\gamma$ must have a removable node $A_{1} \neq A_{0}$. By assumption, $A_{1}$ is connected with both $\mu / \gamma$ and $\nu / \gamma$, and since $[\mu / \gamma]$ is of degree $1, A_{1}$ and $A_{0}$ are the only removable nodes of $\gamma$.

Since $\left[\mu / \gamma_{A_{0}}\right]$ is 1-dimensional, we conclude from Lemmas 4.4 and 4.5 that $\left[\mu / \gamma_{A_{1}}\right]=[n-m, 1]$ or the conjugate. So if $n-m>1$ and $\left[\nu / \gamma_{A_{1}}\right]=[n-m, 1]$ then (3) equals $[n-m, 1] \cdot[n-m, 1]$ or the conjugate. Now we apply Corollary 4.2 and Lemma 4.6. Otherwise $\mu=(k, k), \nu=(k+n-m, k-$ $n+m)$ or $\mu=\left(2^{k}\right), \nu=\left(2^{k-1}, 1^{2}\right)$. But these cases have already been considered. 
Thus we have classified all pairs $\mu, \nu$ such that $[\mu] \cdot[\nu]$ has at most 2 homogeneous components. The "if-parts" of the following conjecture are proved in Corollary 4.2 and Lemma 4.3.

\section{Conjecture.}

(i) $[\mu] \cdot[\nu]$ has 3 homogeneous components if and only if $n=3$ and $\mu=$ $\nu=(2,1)$ or $n=4$ and $\mu=\nu=(2,2)$.

(ii) $[\mu] \cdot[\nu]$ has 4 homogeneous components if and only if one of the following happens:

(a) $n \geq 4$ and $\mu, \nu \in\left\{(n-1,1),\left(2,1^{n-2}\right)\right\}$;

(b) $n=2 k+1$ for some $k \geq 2$, and one of $\mu, \nu$ is in $\left\{(2 k, 1),\left(2,1^{2 k-1}\right)\right\}$ while the other one is in $\left\{(k+1, k),\left(2^{k}, 1\right)\right\}$;

(c) $n=6$ and $\mu, \nu \in\left\{\left(2^{3}\right),\left(3^{2}\right)\right\}$.

The following theorem proves the conjecture in the special case when both $\mu$ and $\nu$ are symmetric.

Theorem 4.9. Let $\mu$ and $\nu$ be symmetric partitions of $n$. Then $[\mu] \cdot[\nu]$ has at most 4 homogeneous components if and only if one of the following holds:

(i) $n=1$;

(ii) $n=3, \mu=\nu=(2,1)$, when $[\mu]^{2}=[3]+[2,1]+\left[1^{3}\right]$;

(iii) $n=4, \mu=\nu=\left(2^{2}\right)$, when $[\mu]^{2}=[4]+\left[2^{2}\right]+\left[1^{4}\right]$.

Proof. Let $\gamma=\mu \cap \nu, m=|\gamma|$. Then $\gamma$ is a symmetric partition, and at least one of the skew diagrams $\mu / \gamma, \nu / \gamma$ has no box on the main diagonal. Say it is $\mu / \gamma$. Because of the symmetry, we can then write $\mu / \gamma$ as a disjoint union $\alpha \cup \alpha^{\prime}$, where $\alpha$ and $\alpha^{\prime}$ are some skew shapes which are conjugate to each other. In particular, $n-m$ is even. By $[6,(5.7)]$,

$$
[\mu / \gamma]=[\alpha] \hat{\otimes}\left[\alpha^{\prime}\right]
$$

If every constituent of $[\alpha] \hat{\otimes}\left[\alpha^{\prime}\right]$ belongs to $M=\left\{[n-m],\left[1^{n-m}\right],[n-\right.$ $\left.m-1,1],\left[2,1^{n-m-2}\right]\right\}$ then by the Littlewood-Richardson Rule, every constituent of $[\alpha]$ and $\left[\alpha^{\prime}\right]$ would have to belong to $\left\{[(n-m) / 2],\left[1^{(n-m) / 2}\right],[(n-\right.$ $\left.m) / 2-1,1],\left[2,1^{(n-m) / 2-2}\right]\right\}$. But even then, if $n-m \geq 6$, the LittlewoodRichardson Rule implies that there are components of $[\alpha] \hat{\otimes}\left[\alpha^{\prime}\right]$ not in $M$.

Assume first that $n-m \geq 6$. Then, by the Littlewood-Richardson Rule again, $[\nu / \gamma]$ contains a constituent not in $M$. Now Theorems 3.4 and $4.8 \mathrm{im}-$ ply that $[\mu / \gamma] \cdot[\nu / \gamma]$ contains at least three different irreducible constituents, say $\left[\hat{\rho_{1}}\right],\left[\hat{\rho_{2}}\right],\left[\hat{\rho_{3}}\right]$. Then $[\mu] \cdot[\nu]$ contains the corresponding constituents $\left[\rho_{1}\right]$, $\left[\rho_{2}\right],\left[\rho_{3}\right]$, thanks to Corollary 2.3. Since $\mu$ and $\nu$ are symmetric, $[\mu] \cdot[\nu]$ also contains the conjugate constituents $\left[\rho_{1}^{\prime}\right],\left[\rho_{2}^{\prime}\right],\left[\rho_{3}^{\prime}\right]$. Now, by Theorem 3.3 no constituent can have at the same time the maximal length and width among all the constituents. Hence $\left[\rho_{i}\right] \neq\left[\rho_{j}^{\prime}\right]$ for all $i, j$. Thus we have found 6 different irreducible constituents. 
The case $n-m=0$ follows from Lemma 4.3. So we may now assume that $n-m=2$ or 4 . Note that in the first case $n>7$ since for $n \leq 7$ there is only one symmetric partition, and in the second case $n>8$, since the intersection of the two different symmetric partitions for $n=8$ is a partition of 6 . Then by the Littlewood-Richardson Rule and Corollary 2.3, we know that $[\mu] \cdot[\nu]$ has the constituents $[n-2,2],\left[n-2,1^{2}\right]$ and their conjugates if $n-m=2$, and it has the constituents $[n-4,3,1]$ and $\left[n-4,2,1^{2}\right]$ and their conjugates if $n-m=4$. By the remark above, $n$ is sufficiently large in both cases so that the four constituents are all different.

Assume that these are all the constituents of $[\mu] \cdot[\nu]$. Consider the case $n-m=4$. We compute the character values on $(n-1)$-cycles and $(n-2)$ cycles. Since $|\gamma|=n-4$, we know that $\min \left(h_{11}^{\mu}, h_{11}^{\nu}\right)<n-2$. Hence on an $(n-1)$-cycle $z_{n-1}$ and an $(n-2)$-cycle $z_{n-2}$ in $S_{n}$ we have by the Murnaghan-Nakayama rule:

$$
[\mu]\left(z_{n-1}\right) \cdot[\nu]\left(z_{n-1}\right)=0=[\mu]\left(z_{n-2}\right) \cdot[\nu]\left(z_{n-2}\right) .
$$

On the other hand, if $n$ is even, then

$$
\left[n-4,2,1^{2}\right]\left(z_{n-1}\right)=-1=\left[4,2,1^{n-6}\right]\left(z_{n-1}\right)
$$

and

$$
[n-4,3,1]\left(z_{n-1}\right)=0=\left[3,2^{2}, 1^{n-7}\right]\left(z_{n-1}\right)
$$

gives a contradiction. If $n$ is odd, then similarly

$$
\left[n-4,2,1^{2}\right]\left(z_{n-2}\right)=0=\left[4,2,1^{n-6}\right]\left(z_{n-2}\right)
$$

and

$$
[n-4,3,1]\left(z_{n-2}\right)=1=\left[3,2^{2}, 1^{n-7}\right]\left(z_{n-2}\right)
$$

gives a contradiction. The case $n-m=2$ is considered similarly using $z_{n}$ and $z_{n-1}$.

\section{Homogeneous Kronecker products of $A_{n}$-representations.}

We first recall the classification of the complex irreducible $A_{n}$-representations (cf. $[4,2.5]$ ). If $\mu$ is a non-symmetric partition of $n$ then the restrictions $S^{\mu} \downarrow_{A_{n}}$ and $S^{\mu^{\prime}} \downarrow_{A_{n}}$ are irreducible and isomorphic to each other. We denote the corresponding irreducible $A_{n}$-module by $T^{\mu}$ or $T^{\mu^{\prime}}$. Thus $T^{\mu} \cong T^{\mu^{\prime}}$ for $\mu \neq \mu^{\prime}$. On the other hand, if $\mu=\mu^{\prime}$ then $S^{\mu} \downarrow_{A_{n}}$ splits into a direct sum of two non-isomorphic $A_{n}$-modules, say $T_{+}^{\mu}$ and $T_{-}^{\mu}$. Moreover, the modules $T_{+}^{\mu}$ and $T_{-}^{\mu}$, as $\mu$ runs over all symmetric partitions of $n$, together with the modules $T^{\mu}$, as $\mu$ runs over a system of representatives of the pairs $\left\{\mu, \mu^{\prime}\right\}$ for the non-symmetric partitions $\mu$ of $n$, form a complete system of the nonisomorphic irreducible $A_{n}$-modules. It is well known that $T_{ \pm}^{\mu}$ is obtained 
from $T_{\mp}^{\mu}$ by twisting with an automorphism of $A_{n}$, which comes from a conjugation by an element $g \in S_{n} \backslash A_{n}$. The character of $T_{( \pm)}^{\mu}$ will be denoted by $\{\mu\}_{( \pm)}$.

Lemma 5.1. Let $\mu, \nu$ be non-symmetric partitions of $n$, both different from (n) and $\left(1^{n}\right)$. Then $T^{\mu} \otimes T^{\nu}$ is homogeneous if and only if $S^{\mu} \otimes S^{\nu} \cong$ $x S^{\lambda} \oplus y S^{\lambda^{\prime}}$ for some $\lambda \neq \lambda^{\prime}, x, y \in \mathbb{N}$.

Proof. This follows from the definition of the modules $T^{\mu}$ and Theorem 3.4.

Lemma 5.2. Let $\mu, \nu$ be partitions of $n$, both different from $(n),\left(1^{n}\right)$. Assume that $\mu \neq \mu^{\prime}, \nu=\nu^{\prime}$. Then $T^{\mu} \otimes T_{ \pm}^{\nu}$ is homogeneous if and only if $S^{\mu} \otimes S^{\nu} \cong x S^{\lambda} \oplus y S^{\lambda^{\prime}}$ for some $\lambda \neq \lambda^{\prime}, x, y \in \mathbb{N}$.

Proof. The "if-part" is clear.

If $T^{\mu} \otimes T_{+}^{\nu} \cong x T_{ \pm}^{\lambda}$ for some $\lambda=\lambda^{\prime}$, then, conjugating by $g \in S_{n} \backslash A_{n}$, we get $T^{\mu} \otimes T_{-}^{\nu} \cong x T_{\mp}^{\lambda}$. So

$$
T^{\mu} \otimes\left(T_{+}^{\nu} \oplus T_{-}^{\nu}\right) \cong x\left(T_{+}^{\lambda} \oplus T_{-}^{\lambda}\right) .
$$

The lift to $S_{n}$ gives $S^{\mu} \otimes S^{\nu} \cong x S^{\lambda}$, which is impossible by Theorem 3.4.

If $T^{\mu} \otimes T_{+}^{\nu} \cong x T^{\lambda}$ for some $\lambda \neq \lambda^{\prime}$, then as above we have $T^{\mu} \otimes T_{-}^{\nu} \cong x T^{\lambda}$, so the lift to $S_{n}$ gives $S^{\mu} \otimes S^{\nu} \cong y S^{\lambda} \oplus z S^{\lambda^{\prime}}$ (with $y+z=x$ ).

Lemma 5.3. Let $\nu$ be a symmetric partition of $n$, and let $\phi, \psi$ be irreducible $A_{n}$-characters both different from $\{\nu\}_{+}$and $\{\nu\}_{-}$. Then

$$
\left\langle\psi \cdot\{\nu\}_{+}, \phi\right\rangle=\left\langle\psi \cdot\{\nu\}_{-}, \phi\right\rangle .
$$

Proof. By $[4,2.5 .13]$, we have

$$
\begin{aligned}
\left\langle\psi \cdot\{\nu\}_{ \pm}, \phi\right\rangle= & \frac{1}{\left|A_{n}\right|} \sum_{g \in A_{n}} \psi(g)\{\nu\}_{ \pm}(g) \overline{\phi(g)} \\
= & \frac{1}{\left|A_{n}\right|}\left(\sum_{g \in A_{n} \backslash\left(C_{\nu}^{+} \cup C_{\nu}^{-}\right)} \psi(g)\{\nu\}_{ \pm}(g) \overline{\phi(g)}\right. \\
& +\sum_{g \in C_{\nu}^{+}} \psi(g) \frac{1}{2}\left(\varepsilon_{\nu} \pm \sqrt{\varepsilon_{\nu} \prod_{i} h_{i i}^{\nu}}\right) \overline{\phi(g)} \\
& +\sum_{g \in C_{\nu}^{-}} \psi(g) \frac{1}{2}\left(\varepsilon_{\nu} \mp \sqrt{\varepsilon_{\nu} \prod_{i} h_{i i}^{\nu}}\right) \overline{\phi(g)}
\end{aligned}
$$

where $\varepsilon_{\nu}=(-1)^{(n-k) / 2}$ and $C_{\nu}^{ \pm}$denote the two conjugacy classes in $A_{n}$ which consist of elements of cycle type $\left(h_{11}^{\nu}, \ldots, h_{k k}^{\nu}\right)$. Since $\psi, \phi$ correspond 
to partitions different from $\nu$, each of them takes the same value on $C_{\nu}^{+}$and $C_{\nu}^{-}$, so the last expression is the same for $\{\nu\}_{+}$and $\{\nu\}_{-}$.

Lemma 5.4. Let $\nu$ be a symmetric partition of $n$ and let $\psi$ be an irreducible $A_{n}$-character different from $\{\nu\}_{+}$and $\{\nu\}_{-}$. Then

$$
\begin{aligned}
& \left\langle\psi \cdot\{\nu\}_{+},\{\nu\}_{+}\right\rangle=\left\langle\psi \cdot\{\nu\}_{-},\{\nu\}_{-}\right\rangle \quad \text { and } \\
& \left\langle\psi \cdot\{\nu\}_{+},\{\nu\}_{-}\right\rangle=\left\langle\psi \cdot\{\nu\}_{-},\{\nu\}_{+}\right\rangle .
\end{aligned}
$$

Proof. We compute the scalar products using $[4,2.5 .13]$ as in the previous proof, and use the facts that $\{\nu\}_{+}(g)=\{\nu\}_{-}(g)$ for any $g \in A_{n} \backslash\left(C_{\nu}^{+} \cup C_{\nu}^{-}\right)$ and $\psi(g)=\psi(h)$ for any $g, h \in C_{\nu}^{+} \cup C_{\nu}^{-}$.

From the previous two results we deduce:

Proposition 5.5. Let $\mu, \nu$ be symmetric partitions of $n, \mu \neq \nu$. Then $\{\mu\}_{+} \cdot\{\nu\}_{+}$is homogeneous if and only if $\{\mu\}_{+} \cdot\{\nu\}_{-}$is homogeneous.

Now we can classify the homogeneous Kronecker products of irreducible $A_{n}$-characters. Note that if $n>4$ then the only 1-dimensional character is the trivial one. For $n=3$ and 4 there are two more 1-dimensional characters in each case: $\{2,1\}_{ \pm}$and $\left\{2^{2}\right\}_{ \pm}$.

Theorem 5.6. Let $\phi, \psi$ be irreducible $A_{n}$-characters both of degrees greater than 1. Then $\phi \cdot \psi$ is homogeneous if and only if $n=a^{2}$ for some $a>2$ and one of the characters is $\{n-1,1\}$, while the other is $\left\{a^{a}\right\}_{+}$or $\left\{a^{a}\right\}_{-}$. In the exceptional case:

$$
\{n-1,1\} \cdot\left\{a^{a}\right\}_{ \pm}=\left\{a+1, a^{a-2}, a-1\right\} .
$$

Proof. The "if-part" follows from Corollary 4.2(ii).

Let $\phi$ and $\psi$ correspond to partitions $\mu$ and $\nu$, respectively. If $\mu$ and $\nu$ are both non-symmetric, then by Lemma 5.1 and Theorem 4.8 the tensor product $T^{\mu} \otimes T^{\nu}$ is not homogeneous. If one of the partitions $\mu, \nu$ is symmetric and the other is not, use Lemma 5.2 and Theorem 4.8. So we may assume that $\mu$ and $\nu$ are both symmetric. If $\mu \neq \nu$, then by Lemmas 5.3, 5.4 and 5.5, if one of the four products $\{\mu\}_{ \pm} \cdot\{\nu\}_{ \pm}$is homogeneous then the product $[\mu] \cdot[\nu]$ has at most two homogeneous components, contradicting Theorems 3.4 and 4.8. Indeed, consider for example the case where $\{\mu\}_{-} \cdot\{\nu\}_{-}$is homogeneous. Since $\{\lambda\}_{ \pm}$is obtained from $\{\lambda\}_{\mp}$ by conjugating with an element $g \in S_{n} \backslash A_{n}$, we conclude that $\{\mu\}_{+} \cdot\{\nu\}_{+}$is also homogeneous. Moreover, if $\{\mu\}_{-} \cdot\{\nu\}_{-}=x\{\lambda\}$ then $\{\mu\}_{+} \cdot\{\nu\}_{+}=x\{\lambda\}$, and if $\{\mu\}_{-} \cdot\{\nu\}_{-}=x\{\kappa\}_{ \pm}$then $\{\mu\}_{+} \cdot\{\nu\}_{+}=x\{\kappa\}_{\mp}$. By Proposition 5.5, we also have that $\{\mu\}_{ \pm} \cdot\{\nu\}_{\mp}$ are homogeneous. Moreover, Lemmas 5.3, 5.4

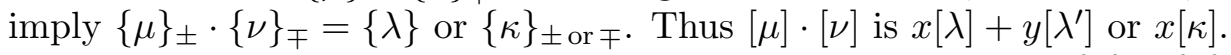

Now let $\mu=\nu$ be symmetric. We have to consider three cases: $\{\mu\}_{ \pm} \cdot\{\mu\}_{ \pm}$ and $\{\mu\}_{+} \cdot\{\mu\}_{-}$. Using conjugation with $g \in S_{n} \backslash A_{n}$, we can eliminate one 
of them, and work only with $\{\mu\}_{+} \cdot\{\mu\}_{+}$and $\{\mu\}_{+} \cdot\{\mu\}_{-}$. Let us consider the first case (the second one is similar). So let $\{\mu\}_{+} \cdot\{\mu\}_{+}=x \psi$ for some irreducible $A_{n}$-character $\psi$.

If the dual character $\{\mu\}_{+}^{*}$ is equal to $\{\mu\}_{+}$, then

$$
\left\langle\{n\},\{\mu\}_{+} \cdot\{\mu\}_{+}\right\rangle=\left\langle\{\mu\}_{+},\{\mu\}_{+}\right\rangle=1,
$$

so we deduce $\{\mu\}_{+} \cdot\{\mu\}_{+}=\{n\}$, which is impossible as $\{\mu\}$ is not of degree 1 .

If $\{\mu\}_{+}^{*}=\{\mu\}_{-}$, then

$$
\left\langle\{n\},\{\mu\}_{+} \cdot\{\mu\}_{+}\right\rangle=\left\langle\{\mu\}_{-},\{\mu\}_{+}\right\rangle=0
$$

and

$$
\begin{aligned}
\left\langle\{n-1,1\},\{\mu\}_{+} \cdot\{\mu\}_{+}\right\rangle & =\left\langle\{n\}+\{n-1,1\},\{\mu\}_{+} \cdot\{\mu\}_{+}\right\rangle \\
& =\left\langle\{n-1\} \uparrow A_{n},\{\mu\}_{+} \cdot\{\mu\}_{+}\right\rangle \\
& =\left\langle\{\mu\}_{-} \downarrow_{A_{n-1}},\{\mu\}_{+} \downarrow_{A_{n-1}}\right\rangle .
\end{aligned}
$$

Consider the case where $\mu$ is not a square. Then, by the Branching Rule, both restrictions in the last expression contain some $\{\lambda\}$ where $\lambda$ is a nonsymmetric partition of $n-1$. So the scalar product above is non-zero, whence $\{\mu\}_{+} \cdot\{\mu\}_{+}=x\{n-1,1\}$. Take $z \in A_{n}$ of cycle type $(n-2,2)$, if $n$ is even and of cycle type $(n-2,1,1)$, if $n$ is odd. As $\mu$ is symmetric it does not have a hook of length $n-2$. Hence by $[4,2.5 .13]$ and the Murnaghan-Nakayama Rule we have

$$
\{\mu\}_{+}(z)\{\mu\}_{+}(z)=0 .
$$

On the other hand, $x\{n-1,1\}(z)= \pm x \neq 0$, when $n$ is odd or even, respectively. This is a contradiction.

It remains to deal with the case where $\{\mu\}_{+}^{*}=\{\mu\}_{-}$and $\mu$ is a square. Consider

$$
\left\langle\{n-2\} \uparrow^{A_{n}},\{\mu\}_{+} \cdot\{\mu\}_{+}\right\rangle=\left\langle\{\mu\}_{-} \downarrow_{A_{n-2}},\{\mu\}_{+} \downarrow_{A_{n-2}}\right\rangle .
$$

By the Branching Rule, the last scalar product is non-zero. But

$$
\{n-2\} \uparrow^{A_{n}}=\{n\}+2\{n-1,1\}+\{n-2,2\}+\left\{n-2,1^{2}\right\},
$$

and the product $\{\mu\}_{+} \cdot\{\mu\}_{+}$can not be of the form $x\{n\}$ or $x\{n-1,1\}$ by the same arguments as before. So we may assume that

$$
\{\mu\}_{+} \cdot\{\mu\}_{+}=x\{n-2,2\} \quad \text { or } \quad\{\mu\}_{+} \cdot\{\mu\}_{+}=x\left\{n-2,1^{2}\right\} .
$$

In the first case, we evaluate both sides on an element of cycle type $\left(n-2,1^{2}\right)$ if $n$ is odd, and on an element of cycle type $(n-1,1)$ if $n$ is even. Then the left hand side gives zero whereas the right hand side is $\pm x$, giving a contradiction.

In the second case, we evaluate both sides on an element of cycle type $(n)$ if $n$ is odd, and on an element of cycle type $\left(n-3,1^{3}\right)$ if $n$ is even. This gives zero on the left hand side and $\pm x$ on the right hand side. 
Acknowledgement. We are grateful to the Mathematical Sciences Research Institute, University of Oregon and the Isaac Newton Institute where the research for this paper was done. The authors were also supported by the NSF (grants \# DMS 9022140 and \# DMS-9600124) and the Deutsche Forschungsgemeinschaft (grant Be 923/6-1).

\section{Note added in proof.}

After this paper had been accepted we learned of the paper of I. Zisser "Irreducible products of characters in $A_{n}$ ", Israel J. Math., 84 (1993), 147151. The main result of the Zisser's paper is that $A_{n}$ has a pair of non-linear characters, whose product is irreducible, if and only if $n$ is a perfect square. Even though Zisser does not classify all such pairs (which is done in our paper), he does prove that one of the characters must correspond to the square diagram. Moreover, he also proves that the product of two non-linear $S_{n^{-}}$ characters is never irreducible, using his previous results on decomposing the squares of irreducible characters. However, we believe that the short direct proof of the more general fact that such a product is never homogeneous given in Section 3 of our paper (Theorem 3.4) might be useful. Generally, our approach allows us to consider more general questions concerning few homogeneous components rather than few irreducible components.

\section{References}

[1] M. Clausen and H. Meier, Extreme irreduzible Konstituenten in Tensordarstellungen symmetrischer Gruppen, Bayreuther Math. Schriften, 45 (1993), 1-17.

[2] Y. Dvir, On the Kronecker product of $S_{n}$ characters, J. Algebra, 154 (1993), 125-140.

[3] A.M. Garsia and J. Remmel, Shuffles of permutations and the Kronecker product, Graphs and Combin., 1 (1985), 217-263.

[4] G. James and A. Kerber, The representation theory of the symmetric group, AddisonWesley, London, 1981.

[5] D.E. Littlewood, The Kronecker product of symmetric group representations, J. London Math. Soc., 31 (1956), 89-93.

[6] I.G. Macdonald, Symmetric functions and Hall polynomials, 2nd edition, Oxford Univ. Press, Oxford, 1995.

[7] F.D. Murnaghan, The analysis of the Kronecker product of irreducible representations of the symmetric group, Amer. J. Math., 60 (1938), 761-784.

[8] J. Remmel, A formula for the Kronecker products of Schur functions of hook shapes, J. Algebra, 120 (1989), 100-118.

[9] J. Remmel and T. Whitehead, On the Kronecker product of Schur functions of two row shapes, Bull. Belg. Math. Soc., 1 (1994), 649-683.

[10] J. Saxl, The complex characters of the symmetric groups that remain irreducible in subgroups, J. Algebra, 111 (1987), 210-219.

[11] E. Vallejo, On the Kronecker product of irreducible characters of the symmetric group, preprint, 1997. 
[12] I. Zisser, The character covering numbers of the alternating groups, J. Algebra, 153 (1992), 357-372.

Received December 15, 1997 and revised July 1, 1998.

Otto-von-Guericke-Universität MagdeburG

D-39016 MAGDEBURG

GERMANY

E-mail address: bessen@mathematik.uni-magdeburg.de

UNIVERSITY OF OREGON

Eugene, Oregon, 97403

E-mail address: klesh@darkwing.uoregon.edu 